Bundesgesundheitsbl - Gesundheitsforsch Gesundheitsschutz 2004 · 47:801-808

DOI 10.1007/s00103-004-0876-6

๑) Springer-Verlag 2004
U. Marcus · Robert Koch-Institut, Berlin

\section{Retroviruskonferenz in San Francisco, 8. bis 11. Februar 2004}

\author{
Teil 1: Epidemiologie, Risiko- und Schutzfaktoren, \\ HIV-Primär- und Superinfektionen, Mutter-Kind-Übertragung
}

Die zu Beginn eines jeden Jahres in den USA stattfindende Retroviruskonferenz (Conference on Retroviruses and 0pportunistic Infections - CROI) gehört zu den größten und wichtigsten internationaIen AIDS-Konferenzen. Schwerpunkte auf der Konferenz sind traditionsgemäß Fragen der Behandlung, der Medikamenten- und Vakzineentwicklung und der virologischen Grundlagenforschung. Nachfolgend berichten wir über ausgewählte Aspekte aus den Bereichen Epidemiologie und Therapie.

\section{Epidemiologie der HIV-Infektion und Risikofaktoren}

In den meisten westlichen Industriestaaten ist in den letzten Jahren eine deutliche Zunahme sexuell übertragbarer Infektionen (STI), insbesondere der Syphilis, bei Männern mit gleichgeschlecht- lichen Sexualkontakten (MSM) zu verzeichnen. In den 29 US-Bundesstaaten, in denen eine Meldung von HIV-Erstdiagnosen bereits etabliert ist, wird seit 1999 ein signifikanter Anstieg der Zahl der HIV-Diagnosen um ca. $5 \%$ pro Jahr bei MSM registriert. Dieser Anstieg ist in allen ethnischen Gruppen zu beobachten (Weiße, Afroamerikaner und Hispanoamerikaner) [Hall et al. Abstr. 86]. Auf den ersten Blick scheint die Interpretation auf der Hand zu liegen: Zunehmendes sexuelles Risikoverhalten bei MSM führt zu einer Zunahme sexuell übertragbarer Infektionen inklusive der HIV-Infektion. Zusätzlich begünstigt eine höhere Verbreitung sexuell übertragbarer Infektionen über die Kofaktorenrolle von Genitalinfektionen die Übertragung von HIV. Bei genauerer Betrachtung der Daten tauchen allerdings Zweifel an einer solchen einfachen Kausalkette auf. In San Francisco wurden die Trends der Syphilis-Inzi- denz mit der HIV-Inzidenz bei MSM verglichen, die von HIV-Testangeboten $\mathrm{Ge}$ brauch machten. Die Inzidenz wurde mithilfe eines Testalgorithmus bestimmt, bei dem durch Testung in unterschiedlich empfindlichen Antikörpernachweissystemen kürzlich erfolgte HIV-Infektionen (im letzten halben Jahr) identifiziert werden können. Stellt man die beiden Inzidenzen einander gegenüber, lässt sich bei MSM von 1998-2002 eine Steigerung der Syphilis-Inzidenz um ca. 1.000\% feststellen, während die HIV-Inzidenz nahezu unverändert bleibt (• Tabelle 1) [Buchacz et al. Abstr. 88].

Auch in der großen Impfstudie, in der zwischen 1998 und 2002 die Wirksamkeit des rekombinanten HIV-Hüllproteins gp120 zur Verhütung einer HIV-Infektion geprüft wurde und an der in den USA knapp 4.700 HIV-negative MSM mit erhöhtem Infektionsrisiko teilnahmen, wurde in diesem Zeitraum keine we-

Tabelle 1

HIV-Inzidenzen bei testwilligen Männern mit gleichgeschlechtlichen Sexualkontakten (MSM) in San Francisco, 1998-2002

\begin{tabular}{|cclll}
\hline Jahr & $\begin{array}{l}\text { Syphilis-Inzidenz/ } \\
100.000\end{array}$ & $\begin{array}{l}\text { Anzahl HIV-Teste } \\
\text { bei MSM }\end{array}$ & $\begin{array}{l}\text { Anzahl HIV- } \\
\text { Neuinfektionen }\end{array}$ & $\begin{array}{l}\text { HIV-Inzidenz in \% in den } \\
\mathbf{2} \text { untersuchten Testeinrichtungen }\end{array}$ \\
\hline 1998 & 8 & 3.517 & 40 & $1,86 / 4,83$ \\
\hline 1999 & 26 & 2.466 & 49 & $3,91 / 5,44$ \\
\hline 2000 & 71 & 3.045 & 41 & $2,68 / 3,34$ \\
\hline 2001 & 223 & 3.036 & 36 & $2,76 / 2,46$ \\
\hline 2002 & 512 & 2.824 & 42 & $2,41 / 4,03$ \\
\hline
\end{tabular}


Tabelle 2

HIV-Inzidenz bei Teilnehmern einer HIV-Impfstudie in den USA, 1998-2002

\begin{tabular}{|lcl|}
\hline Jahr & $\begin{array}{c}\text { Anzahl Serokonversionen/ } \\
\text { Anzahl teilnehmender MSM }\end{array}$ & HIV-Inzidenz/100 Personenjahre \\
\hline $1998-1999$ & $78 / 4.697$ & 2,6 \\
\hline 2000 & $135 / 4.202$ & 3,2 \\
\hline 2001 & $101 / 3.926$ & 2,7 \\
\hline 2002 & $22 / 3.051$ & 1,7 \\
\hline
\end{tabular}

Tabelle 3

Anteil von GBV-C-virämischen,

HIV-koinfizierten Patienten an

den verschiedenen klinische

Stadien der HIV-Infektionen gemäß

der CDC-Klassifikation

\begin{tabular}{|lll}
\hline & HIV & GBV-C-RNA+ \\
\hline CDCI & $38(19 \%)$ & 13 \\
\hline CDC II & $81(41 \%)$ & 19 \\
\hline CDC III & $78(40 \%)$ & 1 \\
\hline
\end{tabular}

sentliche Veränderung der HIV-Inzidenz festgestellt (॰ Tabelle 2) [Ackers et al. Abstr. 857]. Eine erhöhte HIV-Inzidenz wurde in dieser Impfstudie jedoch bei Personen mit Diagnose einer Syphilis, Gonorrhö oder Chlamydieninfektion sowie bei Männern, die Drogen wie Amphetamine, Halluzinogene oder Poppers konsumierten, festgestellt.

Ein Faktor, der zu einer geringen oder fehlenden Steigerung der HIV-Inzidenz bei Zunahme von Risikoverhalten beitragen könnte, ist eine durch antiretrovirale Behandlung reduzierte Infektiosität. Anhand von Risikoverhaltensangaben und beobachteten Serokonversionen in der San Francisco Young Men's Health Study wurde versucht, den Einfluss der Einführung hochaktiver antiretroviraler Therapie auf die Infektiosität in einer Modellrechnung darzustellen. Trotz eines erhöhten Risikoverhaltens (Zunahme der Zahl ungeschützter Sexualkontakte) nach Einführung der HAART wurden in der Studie weniger Serokonversionen beobachtet als vorher, was als Indiz für eine verminderte Infektiosität gewertet wurde. Unter den für die Modellrechnung verwendeten Parametern ergab sich eine Verminderung der Infektiosität um ca. 60\% [1].

Ein Aspekt, der die Diskrepanz zwischen der Zunahme von STIs und HIV teilweise erklären könnte, ist eine Tendenz, Sexualpartner nach deren HIV-Serostatus auszuwählen. Bei der Analyse der Risikoverhaltensangaben von MSM, die anonyme HIV-Testberatungen in San Francisco in den Jahren 1998-2002 aufsuchten, stellten Truong et al. [Abstr. 843] fest, dass der Anteil der Männer, die angaben, in den letzten 12 Monaten Sex mit einem bekannt HIV-positiven Partner gehabt zu haben, von 35\% im Jahre 1998 auf $21,5 \%$ im Jahre 2002 zurückging. Der Anteil derjenigen, die ungeschützten Analverkehr mit einem HIV-positiven Partner hatten, änderte sich allerdings nur geringfügig (von 9,9\% im Jahr 1998 auf $8 \%$ im Jahr 2002).

Ein weiterer Aspekt ist die im Vergleich zu HIV einfachere Übertragbarkeit der klassischen STIs. Am San Francisco General Hospital wurde gemäß den CDC-Empfehlungen im Frühjahr 2003 ein regelmäßiges Screening auf Syphilis, Gonorrhö und Chlamydien bei HIV-positiven Patienten eingeführt. Auf Gonorrhö wurde routinemäßig nicht nur urethral, sondern auch rektal und pharyngeal, auf Chlamydien neben der urethralen Untersuchung regelmäßig auch rektal untersucht. Im Beobachtungszeitraum wurden 814 beschwerdefreie HIV-infizierte Patienten gescreent. Dabei fanden sich $15(1,8 \%)$ frische Syphilisinfektionen. Die meisten Gonorrhö-Infektionen wurden pharyngeal, die meisten Chlamydien-Infektionen rektal entdeckt (zur Verteilung der Infektionen im Hinblick auf die Manifestationsorte siehe - Abb.1) [Klausner et al. Abstr. 889]. Dies ist ein deutlicher Hinweis darauf, dass genital-orale Sexualpraktiken für die Übertragung dieser bakteriellen STIs eine erhebliche Bedeutung haben und dass HIV-Infizierte beim Analverkehr eher die rezeptive Rolle übernehmen.
Trotzdem gibt es natürlich Hinweise auf eine epidemiologische Synergie zwischen sexuell übertragbaren Infektionen und HIV-Neudiagnosen (-Neuinfektionen?). In Dublin, wo sich die Syphilis wie in anderen westeuropäischen Großstädten in den letzten Jahren unter MSM ausgebreitet hat, wurde im Jahr 2000 bei $2 \%$ der HIV-Erstdiagnosen bei MSM gleichzeitig eine Syphilis diagnostiziert, im Jahr 2001 bereits bei $26 \%$, 2002 bei $29 \%$ und bis September 2003 bei $23 \%$. Der Anteil der Syphilispatienten mit bereits bekannter HIV-Infektion liegt in Dublin bei 12\% [Devitt et al. Abstr. 848]. In London wurden an einem Behandlungszentrum im Zeitraum von 1999-2003 frisch erworbene HIV-Infektionen klinisch, epidemiologisch und molekularepidemiologisch näher charakterisiert. 83\% der 125 frischen HIV-Infektionen wurden bei MSM diagnostiziert. Bei $12 \%$ war ein primär resistentes Virus nachweisbar. 25\% der Fälle $(n=32)$ waren 14 Übertragungsclustern zuzuordnen. Die Cluster umfassten jeweils 5 (1), 3 (2) und 2 (11) Fälle. In einem hohen Prozentsatz wurden gleichzeitig mit der HIV-Primärinfektion andere bakterielle STIs diagnostiziert. Besonders hoch war der Prozentsatz der STI-Koinfektionen in den Übertragungsclustern (36\% vs. $25 \%$ ) [Pao et al. Abstr. 392]. In einer anderen Studie aus Großbritannien wurden bei 2 Gruppen nicht antiretroviral behandelter, HIV-infizierter MSM die HIV-Plasmakonzentration und die HIVKonzentration im Ejakulat bestimmt. Die eine Gruppe bestand aus Männern mit einer symptomatischen Urethritis, die andere aus Männern ohne Genitalinfektionen. Die HIV-Konzentration im Plasma und die CD4-Zellzahl waren in beiden Gruppen vergleichbar. Im Ejakulat war die HIV-Viruslast bei Männern mit urethraler Gonokokken- und Chlamydieninfektion im Mittel um 0,7 log höher als in der Kontrollgruppe, während bei Männern mit unspezifischer Urethritis die genitale Viruslast nicht erhöht war. Nach Antibiotikabehandlung ging die genitale Viruslast zurück [Sadiq et al. Abstr. 458].

Mithilfe des routinemäßigen Einsatzes von HIV-PCR-Testen in HIV-Testund Beratungsstellen wurde in North Carolina versucht, traditionelle Strategien der STI-Kontrolle wie Partnersuche und 
Behandlung von frisch Infizierten auch für die Kontrolle der HIV-Ausbreitung nutzbar zu machen. Die erheblichen Kosten für die routinemäßige HIV-PCR-Testung wurden durch das Poolen der Proben auf ca. 2 US\$ pro untersuchter Probe reduziert. Zwischen November 2002 und Oktober 2003 wurden knapp 110.000 Proben untersucht, davon waren 622 HIV-positiv. Von den 622 positiven Proben wurden $21(4,1 \%)$ nur durch die PCR entdeckt, bei weiteren 121 deuten die Ergebnisse der Untersuchung mit unterschiedlich sensitiven HIV-Testen (STARHS-Testung) auf eine im letzten halben Jahr erfolgte Infektion hin. Besonders hoch war die Rate PCR-positiver, Antikörper-negativer Proben in Gefängnissen $(11,1 \%)$ und STD-Kliniken $(6,4 \%)$ [Pilcher et al. Abstr. 20]. Die dem Infektionszeitpunkt nahe Diagnose der HIV-Infektion erleichtert die Suche nach der möglichen Infektionsquelle. Sinnvoll, aber politisch in den USA offenbar nicht durchsetzbar, wäre natürlich auch eine Verminderung von Ansteckungsrisiken in Gefängnissen z. B. durch Spritzenaustauschprogramme.

Verschiedene Studien beschäftigen sich mit der Frage, ob und inwiefern die Durchführung einer antiretroviralen Behandlung und/oder eine niedrige Viruslast Auswirkungen auf das Risikoverhalten von HIV-Infizierten haben. Diamond et al. [Abstr. 847] stellten bei der Untersuchung von 874 zufällig ausgewählten $\mathrm{Pa}$ tienten von 6 HIV-Behandlungseinrichtungen in Kalifornien fest, dass bei univariater Analyse sowohl die Durchführung einer antiretroviralen Therapie als auch eine Viruslast unterhalb der Nachweisgrenze mit einer geringeren Häufigkeit ungeschützter Sexualkontakte korreliert sind. Außerdem geht eine bessere Adhärenz auch mit einer geringeren Tendenz zu unsafen Sexualkontakten einher. Allerdings werden diese Zusammenhänge bei multivariater Analyse durch eine Reihe weiterer Faktoren wie Gebrauch von Drogen und Anzahl der Sexualpartner abgeschwächt. Zu ähnlichen Ergebnissen gelangte eine Gruppe aus San Francisco, die sich auf das Risikoverhalten von HIV-Infizierten mit nachgewiesen resistenten Viren konzentriert [ChinHong et al. Abstr. 845]. Die Wissenschaftler finden keine Unterschiede im Risiko-

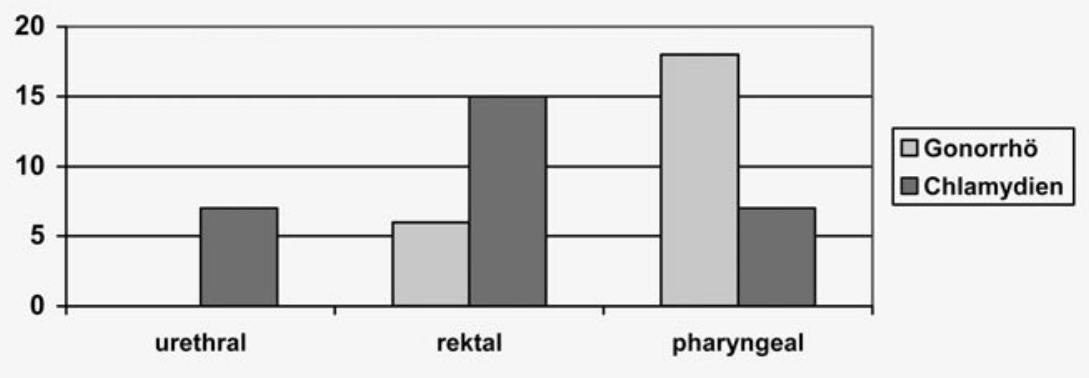

Abb.1 Manifestationsorte von Gonorrhö und Chlamydieninfektionen, entdeckt bei Screeninguntersuchungen von symptomlosen HIV-Infizierten

verhalten zwischen HIV-Infizierten mit resistenten Viren sowie nachweisbarer Viruslast und mit Infizierten mit nachweisbarem Wildtypvirus bzw. Infizierten mit einer Viruslast unterhalb der Nachweisgrenze. Als Prädiktoren für ungeschützten Anal- oder Vaginalverkehr mit HIVnegativen oder ungetesteten Partnern identifizieren sie jüngeres Alter $(<35 \mathrm{Jah}$ re), Depressionen, Gebrauch von Drogen und geringeres Bildungsniveau.

Bekannte Faktoren, die die Empfänglichkeit von Frauen für eine sexuell übertragene HIV-Infektion beeinflussen, sind die hormonellen Einflüssen unterworfene Dicke des vaginalen Schleimhautepithels und genitale Infektionen. Im Rhesusaffenmodell konnten Smith et al. [Abstr. 886] zeigen, dass durch eine Lokalbehandlung mit Östradiol-Salbe das Vaginalepithel dicker und undurchlässiger und damit für SIV weniger empfänglich wird. Die lokale Östradiol-Salbenbehandlung könnte daher eine gute Ergänzung zur - zukünftig hoffentlich möglichen Mikrobizidbehandlung darstellen.

In einem Zellkulturmodell fanden Patterson et al. [Abstr. 885], dass die HIVEmpfänglichkeit von zervikalem Gewebe bei Frauen mit Trichomonas-Infektion durch Behandlung mit zervikovaginaler Spülflüssigkeit gesteigert wird. Sie führen diesen Effekt auf eine Stimulierung der TNF- $\alpha$-Produktion durch die Spülflüssigkeit zurück und propagieren daher ein aggressiveres Screening auf Trichomonaden und deren prompte Behandlung. In einer in Uganda durchgeführten epidemiologischen Studie wurde untersucht, ob eine bakterielle Vaginose eher als Ursache oder als Folge einer HIV-Infektion anzusehen ist. In der Längsschnittstudie an 4.244 Frauen konnte eine bakterielle Vaginose nicht als Risikofaktor für den
Erwerb einer HIV-Infektion identifiziert werden, aber die Prävalenz der bakteriellen Vaginose nahm mit der HIV-Infektion und fortschreitendem Immundefekt zu [Gray et al. Abstr. 146].

\section{Schutzfaktoren}

Ein schon länger bekanntes, aber noch unzureichend verstandenes Phänomen sind Schutzmechanismen, die bei einigen klar HIV-exponierten Personen das Angehen einer HIV-Infektion verhindern. Im Rahmen einer Studie in Seattle konnte mit hochempfindlichen PCR-Testen bei 12 von 94 solcher Personen nichtreplizierendes HIV in Blutzellen nachgewiesen werden. Im Verlauf der Studie serokonvertierten allerdings 10 Patienten. Bei 4 dieser Serokonverter, die einen HIV-positiven festen Partner hatten, wurde das Post-Serokonversionsvirus mit dem Virus des Partners verglichen. Überraschenderweise zeigte sich, dass sich die zur Serokonversion führenden Viren vom Partnervirus deutlich unterschieden. Dies deutet darauf hin, dass die bei exponierten, aber seronegativ bleibenden Personen feststellbaren Immunantworten zwar vor dem Partnervirus schützen, aber nicht vor anderen Virusvarianten [Zhu et al. Abstr. 25].

\section{Schützt eine Infektion mit dem GBV-C-Virus vor einer schnellen Progression der HIV-Erkrankung?}

GBV-C ist ein dem Hepatitis-C-Virus verwandtes Flavivirus, das 1995/96 bei Patienten mit Hepatitis Non A-E entdeckt wurde. Die anfängliche Vermutung, dass es für Non-A-E-Hepatitiden verantwortlich sein könnte (das Virus wurde kurze Zeit auch als Hepatitis-G-Virus bezeichnet), 
konnte nicht bestätigt werden. Bislang ist keine Assoziation zwischen GBV-C und bestimmten Erkrankungen beim Menschen nachweisbar. Die Übertragung des Virus kann parenteral, sexuell und vertikal erfolgen. Bei ca. 60-75\% der Infizierten kommt es zu einer Viruselimination durch das Immunsystem, danach zeigt der Nachweis von Anti-E2-Antikörpern gegen das sog. E2-Hüllprotein die erfolgte Auseinandersetzung mit dem Virus an. Bei $25-40 \%$ der Infizierten verläuft die Infektion chronisch mit Persistenz der GBVC-RNA im Plasma. GBV-C repliziert u. a. in mononukleären Zellen des peripheren Blutes, d. h. auch in CD4+-Zellen.

1998 erschien die erste Publikation [2], die GBV-C als günstigen Prognoseparameter bei HIV-Infizierten beschrieb. Die Ergebnisse der deutschen Arbeitsgruppe wurden durch Publikationen einer französischen [3], japanischen [4] und amerikanischen Gruppe [5] bestätigt, während aus Schweden [6], den USA [7] und Großbritannien [8] widersprechende Ergebnisse veröffentlicht wurden. In der Erstpublikation wurden 197 HIV-infizierte Patienten [IVDA (i.v.-Drogenabhängigen) und MSM], die zwischen Januar 1993 und Dezember 1994 in der Infektionsambulanz der Medizinischen Hochschule Hannover betreut wurden, auf eine GBV-C-Koinfektion untersucht. Es wurden 33 „chronisch“ GBV-C Infizierte ermittelt (positiver RNA-Nachweis). Die GBV-C-Infizierten verteilten sich folgendermaßen auf die Gesamtzahl der HIV-Infizierten, stratifiziert nach ihrer Einordnung in die CDC-Klassifikation (• Tabelle 3).

Überlebenszeitanalysen zeigten für GBV-C-RNA-positive Personen einen signifikanten Überlebensvorteil gegenüber GBV-C-anti-E2-AK-positiven und nicht GBV-C-exponierten Patienten (hazard ratio 0,22). Diese Ergebnisse wurden durch weitere Publikationen mit unterschiedlichen Patientenkollektiven (z. B. Hämophiliepatienten) bestätigt. In Folgestudien wurde zusätzlich über eine Hemmung der HIV-Replikation in der Zellkultur nach gleichzeitiger Infektion mit GBV-C [9] und über einen Anstieg der GBV-CRNA bei Patienten unter HAART [10] berichtet.

Widersprüchliche Ergebnisse wurden aus Schweden, den USA und Großbritan- nien publiziert. Diese sind z. T. durch Differenzen in der Auswertung der Daten (Vergleich GBV-C-RNA-positiver und Anti-E2-Antikörper-positiver Patienten zusammengefasst gegen nicht GBV-Cexponierte Patienten) erklärbar. Unterschiede zwischen den Studien betreffen u. a. die Kenntnis über den Zeitpunkt der HIV-Serokonversion sowie den Zeitpunkt der Bestimmung des GBV-C-Status. Um diese Widersprüche zu klären, wurde eine Studie am Patientenkollektiv der amerikanischen Multicenter AIDSCohort Study (MACS) durchgeführt. Es handelt sich um eine Longitudinalstudie mit 271 Serokonvertern (eingrenzbares Serokonversionsdatum). Die Bestimmung des GBV-C-Status konnte für eine Teilgruppe $(n=138)$ zu 2 Zeitpunkten erfolgen, die 5-6 Jahre auseinander lagen. Bei der ersten Untersuchung war bei 107 der 271 Teilnehmer der Test auf GBV-C-RNA positiv. Anti-E2-Antikörper als Hinweis auf eine durchgemachte Infektion wurden bei weiteren 124 Teilnehmern nachgewiesen, sodass insgesamt 231 der 271 Teilnehmer mit GBV-C infiziert waren bzw. eine Infektion durchgemacht hatten. Bei den zu 2 Zeitpunkten getesteten Teilnehmern ( $n=138)$ war bei 49 Personen der Test auf GBV-C-RNA beide Male positiv, bei 12 Personen kam es aber zwischen der ersten und zweiten Testung zum Verlust der Positivität. Eine Überlebenszeitanalyse auf Basis des zum ersten Zeitpunkt bestimmten GBV-C-Status zeigte keine signifikanten Unterschiede gegenüber Anti-E2-Antikörper-positiven oder nicht exponierten Patienten. Eine Überlebenszeitanalyse auf Grundlage des zu einem späteren Zeitpunkt bestimmten GBV-C-Status ergab hingegen einen signifikanten Überlebensvorteil für die Gruppe mit dem positiven GBVC-RNA-Nachweis. Die Ursache hierfür ist in erster Linie der deutlich ungünstigere Krankheitsverlauf in der Gruppe der 12 Patienten, die die RNA zwischen Erstund Zweittestung verloren hatten [11]

Mittlerweile gibt es noch 2 weitere Untersuchungen an Langzeit-(Serokonverter-)kohorten [12, 13]. In der niederländischen Studie erfolgte die Testung der ersten Probe auf GBV-C kurz nach der HIVSerokonversion, als zweite Probe wurde die letzte Probe vor 1996 untersucht. Bei
Ersttestung waren 137 Personen GBV-CRNA-positiv, bei 134 Studienteilnehmern waren Anti-E2-Antikörper nachweisbar. Bis zur Zweittestung hatten 79 (58\%) der ursprünglich 137 GBV-C-RNA-positiven Teilnehmer die RNA verloren, 10 waren neu RNA-positiv. Teilnehmer, die die GBV-C-RNA verloren hatten, zeigten ein signifikant höheres AIDS-Risiko als Teilnehmer mit durchgehend negativem GBV-C-RNA-Befund. Es konnte kein verlangsamender Effekt des positiven Anti-E2-Antikörpernachweises oder einer persistierenden GBV-C-RNA auf die Progression der HIV-Erkrankung nachgewiesen werden. Die Hypothese der Autoren des Berichtes ist, dass die Persistenz von GBV-C mit der CD4-Zellzahl assoziiert ist, d.h., dass letztlich der Abfall der CD4-Zellzahl bei der HIV-Progression dazu führen würde, dass sich das GBV-CVirus nicht mehr replizieren kann und die Virus-RNA daher verloren geht. Die Ergebnisse der schwedischen Studie aus Malmö, in der der Krankheitsverlauf von 230 HIV-Infizierten analysiert wurde, weisen in dieselbe Richtung. Bei Studieneinschluss wiesen 62 Patienten eine GBV-CVirämie auf, 69 trugen Anti-E2-Antikörper. Von 44 anfänglich virämischen Patienten verloren 11 das GBV-C-Virus, ohne Anti-E2-Antikörper zu bilden. Im Vergleich zu den anderen 3 Gruppen (persistierende Virämie, Antikörper, weder Antikörper noch Virämie) zeigten diese $11 \mathrm{~Pa}$ tienten den ungünstigsten Verlauf ihrer HIV-Erkrankung. Zur Erklärung der bisher erhobenen Befunde gibt es also bislang 2 Möglichkeiten:

1. Eine produktive Infektion mit GBVC (positiver RNA-Nachweis) besitzt im Hinblick auf die Progression der HIV-Infektion einen protektiven Effekt. Dieser beruht entweder auf einer direkten Virusinteraktion, wie z. B. auf einer gegenseitigen Replikationshemmung, oder auf einer Induktion zellulärer Gene und/oder Zytokine durch das GBV-C, die den Verlauf der HIV-Infektion günstig beeinflussen.

2. Der niedrige Anteil GBV-C-RNApositiver Patienten unter HIV-Infizierten in fortgeschrittenen Stadien ist kein Zeichen für einen protektiven Effekt, sondern eine Folge des Verlustes der GBV-CReplikationsfähigkeit in diesen Stadien, z. B. durch eine Zerstörung der GBV-C- 
Wirtszellen. Vor allem die Beobachtung, dass bei Patienten, die die GBV-C-RNA über die Zeit verlieren, keine Antikörper gegen das virale E2-Hüllprotein nachweisbar sind, spricht gegen ein immunologisches Clearing der Infektion und eher für die Hypothese des Verlustes der Wirtszellen.

\section{Postexpositionsprophylaxe nach sexueller Exposition: wirksam oder nicht?}

In San Francisco wurden die Verlaufsuntersuchungen von 700 Personen ausgewertet, die eine nicht beruflich bedingte medikamentöse Postexpositionsprophylaxe (PEP) erhalten hatten [Roland et al. Abstr. 888]. Innerhalb des Untersuchungszeitraumes von 12 Wochen nach dem Expositionsereignis wurden 7 Serokonversionen (1\%) ermittelt. In einem Fall war bereits in der Ausgangsprobe zu Beginn der PEP Virus nachweisbar. Alle Serokonverter berichteten eine Exposition über ungeschützten rezeptiven Analverkehr, wobei in 4 Fällen der positive HIV-Status des Partners bekannt war, bzw. über weitere potenzielle Expositionen in den 6 Monaten vor der PEP. Drei Serokonverter berichteten über weitere Expositionen nach Ende der PEP. In 2 Fällen wurden die PEP-Medikamente nicht regelmäßig eingenommen. Eine Wirksamkeitsstudie zur nicht beruflich bedingten PEP ist angesichts wiederholter Expositionen und der Schwierigkeiten, die Serokonversion einem bestimmten Ereignis und/oder Partner zuzuordnen, wahrscheinlich extrem schwierig. Aber auch die deutschen Erfahrung mit bislang 3 dokumentierten Serokonversionen nach nicht beruflicher PEP deuten darauf hin, dass die medikamentöse PEP nach sexueller Exposition möglicherweise weniger wirksam ist als vielfach erhofft.

\section{HIV-Primär- und -Superinfektion}

Nach der Beschreibung von Einzelfällen hat die Suche nach HIV-Superinfektionen bei chronisch infizierten Personen keine Anhaltspunkte für eine nennenswerte Anzahl solcher Ereignisse erbracht. Eine Arbeitsgruppe in Südkalifornien untersuchte jetzt diesbezüglich ei- ne Kohorte von 54 neu Infizierten. Die Personen wurden zunächst nicht antiretroviral behandelt. Eine deutliche Veränderung der Virusvarianten als Hinweis auf eine erfolgte Superinfektion wurde bei 3 Personen beobachtet (entspricht einer Rate von 6,5\%/Jahr). Die veränderten Viren traten 5-13 Monate nach der Erstinfektion auf. In 2 Fällen erfolgte die Erstinfektion mit einem medikamentenresistenten Virus, die vermutete Superinfektion durch ein Wildtypvirus. In einem Fall folgte der Infektion mit dem Wildtypvirus eine Infektion mit einem resistenten Virus. Nach der vermuteten Superinfektion stieg die Viruslast in allen 3 Fällen deutlich an, und die T-Helferzellzahl sank [Smith et al. Abstr. 21].

Eine Folge der gleichzeitigen oder aufeinander folgenden Infektionen mit unterschiedlichen Virusvarianten ist ihre Rekombination $\mathrm{zu}$ viralen Mischtypen mit neuen Eigenschaften. Während Rekombinationen zwischen verschiedenen HIV-Subtypen relativ einfach nachgewiesen werden können, ist dies bei Rekombinationen zwischen verschiedenen Varianten desselben Virussubtyps deutlich schwieriger. Virusisolate von 3 Patienten mit frisch erworbenen HIV-Infektionen wurden daher mit speziellen Analyseverfahren untersucht: Ein Patient hatte sich offenbar gleichzeitig mit einer Mischung aus 2 unterschiedlichen Virusvarianten infiziert, d.h., bei diesem Patienten ließen sich Rekombinanten zwischen den beiden Viruspopulationen identifizieren. Bei dem zweiten Patienten legte die Analyse eher eine Superinfektion als eine Koinfektion nahe. Bei diesem Patienten fanden sich keine Hinweise auf eine Rekombination der beiden Viruspopulationen. Beim dritten Patienten erfolgte die Infektion mit einer Virusvariante, die sich im zeitlichen Verlauf diversifizierte. In diesem Fall lagen Hinweise auf Rekombinationsereignisse vor [Palmer et al. Abstr. 455].

Im Rahmen der Multicenter AIDS Cohort Study (MACS) wurden 32 Serokonverter retrospektiv auf Superinfektionen analysiert. Das Vorliegen einer solchen konnte in einem Fall nachgewiesen werden. Sie erfolgte zwischen 0,8 und 1,3 Jahre nach der Serokonversion. Das superinfizierende Virus verdrängte das ursprüng- liche Virus innerhalb von 6 Monaten vollständig. Der nachfolgende Krankheitsverlauf war ungewöhnlich schnell: 2,4 Jahre nach Serokonversion war die CD4-Zellzahl auf unter 200 Zellen/ $\mu$ l gesunken, und 3,4 Jahre nach Serokonversion erkrankte der Patient an AIDS. Es bleibt allerdings unklar, ob die Superinfektion die Ursache für den schnellen Krankheitsverlauf war oder ob bestimmte Wirtsfaktoren, die $\mathrm{zu}$ einem solchen beitragen, auch die Anfälligkeit für eine Superinfektion erhöhen [Gottlieb et al. Abstr. 454]. In 2 zusätzlichen Kohortenstudien wurden 4 weitere Fälle ermittelt, bei denen es sich aber eher um Ko- als um Superinfektionen handelte, da bereits unmittelbar nach der Infektion 2 unterschiedliche Virusvarianten nachweisbar waren [14]. Bei allen Patienten, die mit mehr als einer Virusvariante infiziert worden waren, verlief die HIV-Infektion ungewöhnlich rasch. Die erste gemessene Viruslast lag bei ihnen im Schnitt etwas höher als bei Patienten, die nur mit einer Virusvariante infiziert wurden. Jedoch war sie nicht so ausgeprägt, um den schnelleren Krankheitsverlauf zu erklären. Obwohl die kleine Fallzahl eine weit reichende Verallgemeinerung verbietet, legt diese Beobachtung den Verdacht nahe, dass Infektionen mit mehr als einer Virusvariante die immunologische Infektionskontrolle erschweren, d. h. eine rasche Krankheitsprogression bedingen.

In einem weiteren Fallbericht wurde ein Patient beschrieben, der sich initial mit einem multiresistenten Virus infiziert hatte, das ohne Therapie immunologisch relativ gut kontrolliert wurde (Virus-Setpoint bei 1.000 Viruskopien/ ml). 6 Monate nach der Infektion wurde ein plötzlicher Anstieg der Viruslast bemerkt, der auf eine Superinfektion mit einem Wildtypvirus zurückgeführt werden konnte. Retrospektiv wurde die HIVspezifische CD8-Zellantwort untersucht und analysiert, in welchem Umfang das superinfizierende Virus noch von der zellulären Immunabwehr erkannt werden konnte. In der gag- und nef-Region der beiden Viren wurden Unterschiede festgestellt, die zu einem Nichterkennen des superinfizierenden Virus durch die existierenden CD8-Zellen führten. Interessanterweise ergab ein Vergleich der Replika- 
tionskapazität des ersten und zweiten Virus eine vergleichbare oder sogar leicht niedrigere Replikationskapazität des superinfizierenden Virus. Dies ist ein Hinweis darauf, dass die virale Fitness auch dadurch beeinflusst wird, wie gut das $\mathrm{Vi}$ rus durch die zelluläre Immunantwort erkannt wird [Daar et al. Abstr. 394]. Frühere Befunde, dass sich das Virus der antikörpervermittelten Immunantwort durch eine ständige Veränderung seiner Hülle entzieht, werden durch eine weitere Untersuchung bestätigt [Frost et al. Abstr. 22].

\section{Mutter-Kind-Übertragung von HIV}

Leider wird die Empfehlung, allen Schwangeren im Rahmen der Schwangerschaftsvorsorge einen HIV-Test anzubieten, noch immer nicht durchgehend befolgt. In den USA wurde eine multizentrische Studie durchgeführt, in der Schwangeren, bei denen bei Klinikaufnahme zur Entbindung kein HIV-Testergebnis bekannt war, eine Beratung und ein HIVSchnelltest angeboten wurde [Bulterys et al. Abstr. 95]. Zwischen November 2001 und Juni 2003 wurden 5.374 Frauen registriert, deren HIV-Status nicht dokumentiert war (entspricht 7,8\% aller im Rahmen der Studie registrierten Schwangerschaften). 4.330 Frauen (81\%) wurde der HIV-Schnelltest angeboten, 3.660 (85\%) Frauen akzeptierten das Testangebot. In 29 Fällen fielen der Schnelltest und der anschließende Bestätigungstest positiv aus. Die Sensitivität und Spezifität des Schnelltestes waren außerordentlich hoch (100\% bzw. 99,9\%), der positive Vorhersagewert lag mit 94\% deutlich höher als für einen Standard-EIA-Test (78\%). Das Testergebnis stand für den Schnelltest im Mittel 70 Minuten nach Blutentnahme zur Verfügung, bei Standardtestung im Labor mittels EIA wurden 28 Stunden benötigt. Der HIV-Schnelltest scheint daher eine gute Möglichkeit zu bieten, bei im Rahmen der Schwangerenvorsorge nicht auf HIV getesteten Schwangeren noch unmittelbar vor der Geburt den HIV-Status abzuklären und ggf. noch prophylaktische Maßnahmen zu ergreifen.

Eine intensiv diskutierte Strategie zur Reduktion der Mutter-Kind-Übertragung ist die Ultrakurzprophylaxe mit Ne- virapin. In einer thailändischen Studie wurde eine relativ kurze pränatale Zidovudin-Monoprophylaxe ab Schwangerschaftswoche 34-36 mit einer NevirapinKurzprophylaxe (eine mütterliche Dosis kurz vor Entbindung und eine kindliche Dosis am dritten Lebenstag) sowie einer Zidovudin-Sirup-Prophylaxe beim Neugeborenen über 4 Wochen miteinander kombiniert. Bei 223 auswertbaren Mutter-Kind-Paaren lag die Übertragungsrate mit 10 infizierten Kindern bei $4,5 \%$. Eine Resistenzentwicklung gegen Nevirapin wurde bei $17 \%$ der Mütter, gegen Zidovudin nur bei $2 \%$ der Mütter dokumentiert. Auch bei 2 der 10 infizierten Neugeborenen waren Resistenzmutationen gegen Nevirapin nachweisbar [Chalermchokcharoenkit et al. Abstr. 96]. Bei der mit französischer Unterstützung an der Elfenbeinküste durchgeführten Ditrame-Plus-Studie wurde ebenfalls eine präpartale Zidovudinprophylaxe ( $\mathrm{ab}$ Woche 36) mit einer unmittelbar vor der Geburtseinleitung verabreichten Nevirapin-Dosis kombiniert. 74 Frauen konnten 4 Wochen nach der Geburt einer Resistenztestung unterzogen werden. Bei 28\% der Frauen waren eine oder mehrere Nevirapin-Resistenzmutationen nachweisbar [Chaix et al. Abstr. 657]. Ursache für die hohen Resistenzraten ist zum einen die niedrige genetische Barriere (eine Punktmutation reicht aus), zum anderen die lange Halbwertszeit der Substanz. Nach Gabe einer 200-mg-Dosis ist Nevirapin im Mittel bis zu 2 Wochen lang nachweisbar (11-21 Tage), d. h., die Kurzprophylaxe führt praktisch zu einer mehrtägigen Monotherapie mit entsprechendem Selektionsdruck auf das Virus [Muro et al. Abstr. 891].

Eine nahe liegende Strategie zur Vermeidung der Resistenzentwicklung wäre die vorübergehende Behandlung mit einer Dreifachkombination. In einer irischen Studie wurden 40 Schwangere ohne mütterliche Behandlungsindikation (CD4-Zellzahl >300/ $\mu \mathrm{l}$ ) im letzten Schwangerschaftsdrittel mit einer HAART behandelt, die nach der Entbindung wieder abgesetzt wurde. Bei einer Kombinationstherapie mit Nevirapin wurde es einige Tage vor den anderen Medikamenten abgesetzt. Eine Resistenztestung konnte bei 30 zuvor unbehan- delten Frauen durchgeführt werden. Die Kombination Combivir/Nevirapin war die am häufigsten verwendete. Die virologische Wirksamkeit war sehr gut, nur bei einer Schwangeren lag die Viruslast zum Entbindungstermin über 1.000 Kopien/ml. Dennoch wurden nach der Entbindung und nach Absetzen der HAART bei 5 Frauen (16\%) Resistenzen gegen $\mathrm{Ne}$ virapin nachgewiesen. In Bezug auf die Ausgangsviruslast, Viruslast bei Entbindung, Ausgangs-CD4-Zellzahl und Therapiedauer gab es keine Unterschiede zwischen den Frauen, die eine Resistenz entwickelten, und denen ohne Resistenzentwicklung [Lyons et al. Abstr. 892]. Die klinischen Konsequenzen der Resistenzselektion nach Nevirapin-Kurzprophylaxe sind noch unklar. Die Befürchtung ist, dass die Kurzprophylaxe bei einem erheblichen Anteil der Frauen zu einer reduzierten Wirksamkeit von NNRTIs beitragen könnte, die einen wichtigen Bestandteil der zur Therapie in Entwicklungsländern empfohlenen Kombinationspräparate darstellen.

In einer größeren amerikanischen Studie wurde bei knapp 3.00o Schwangerschaften der Einfluss der antiretroviralen Therapie, des Entbindungsmodus und der Viruslast zum Entbindungszeitpunkt auf die HIV-Übertragungsrate auf das Neugeborene untersucht. In der Gesamtkohorte lag die Übertragungsrate bei 2,9\% (85 Kinder). Das Übertragungsrisiko stieg mit der Höhe der Viruslast zum Entbindungszeitpunkt. Eine Prophylaxe mit 2 oder mehr Substanzen war in der Regel erfolgreicher als eine Monoprophylaxe. Lag die Viruslast unter 1.00o Kopien, war kein Unterschied in der Übertragungsrate zwischen Kaiserschnitt- und vaginaler Entbindung feststellbar, bei höherer Viruslast hatte die Kaiserschnittentbindung jedoch einen protektiven Effekt [Shapiro et al. Abstr. 99]. Ob sich die Höhe der Viruslast nach einer zeitweisen antiretroviralen Prophylaxe in der Schwangerschaft gegenüber den Ausgangswerten vor Prophylaxebeginn verändert, wurde an einem Behandlungszentrum in St. Louis untersucht. In den 60 Fällen, in denen die antiretrovirale Therapie nach der Entbindung wieder abgesetzt wurde, kehrten Viruslast und THelferzellzahl wieder auf das Niveau vor 
der Schwangerschaft zurück [Gerber et al. Abstr. 945].

Nur für wenige Substanzen liegen bislang ausreichende Daten zur Pharmakokinetik bei Schwangeren vor. Diese wurde für Nevirapin bei 12 Schwangeren im zweiten und dritten Trimester nach 4 oder mehr Wochen unter einer Combivir/Nevirapin-Kombination untersucht. Gegenüber den Werten bei Nicht-Schwangeren zeigten sich keine auffälligen Unterschiede, sodass die übliche Dosierung in der Schwangerschaft nicht verändert werden muss [Aweeka et al. Abstr. 932].

Mehrere Studien befassten sich auch mit den Nebenwirkungen einer antiretroviralen Therapie in der Schwangerschaft. In der PACTG-1022-Studie wurde die Verträglichkeit einer Combivir/Nelfinavirmit einer Combivir/Nevirapin-Therapie verglichen. Eine zum Behandlungsabbruch führende Lebertoxizität wurde bei einer von 21 Frauen unter Nelfinavir und bei 3 von 17 Frauen unter Nevirapin beobachtet.Außerdem trat unter Nevirapin noch ein Stevens-Johnson-Syndrom auf. In einem der 3 oben genannten Lebertoxizitätfälle entwickelte sich ein fulminantes Leberversagen, an dem die Patientin verstarb. Alle schweren Nebenwirkungen unter Nevirapin traten bei Schwangeren mit vergleichsweise hohen T-Helferzellzahlen (>250 CD4-Zellen/ $\mu$ l) vor Therapiebeginn auf [Hitti et al. Abstr. 938]. Auf die erhöhte Nevirapin-Toxizität bei höheren CD4-Zellzahlen wird mittlerweile auch in den entsprechenden Fachinformationen hingewiesen. Im Unterschied dazu wurde bei einem Vergleich der Verträglichkeit von Nevirapin bei schwangeren und nicht-schwangeren Frauen eine deutlich bessere Verträglichkeit bei einem Nevirapin-Behandlungsbeginn in der Schwangerschaft festgestellt [Bershoff-Matcha et al. Abstr. 939].

Bei der Durchsicht von Meldungen an das Nebenwirkungsregister der FDA wurde häufig über eine Lebertoxizität von Substanzen nach antiretroviraler Behandlung von Schwangeren berichtet. Über ein Leberversagen wurde in 18 Fällen berichtet, in 6 Fällen mit tödlichem Ausgang (3-mal ZDV+3TC+NVP, 2-mal $\mathrm{ddI}+\mathrm{d} 4 \mathrm{~T}+\mathrm{NVP}$, $\left.1-\mathrm{mal} \mathrm{ddI}+\mathrm{d}_{4} \mathrm{~T}+\mathrm{NFV}\right)$. Am häufigsten fanden sich erhöhte Transaminasewerte (64), gefolgt von erhöh- ten Cholestasewerten (34), HELLP-Syndrom (27) und Laktatazidose (21). Die am häufigsten als verursachend angesehenen Substanzen waren Lamivudin (35), Zidovudin (34) und Nevirapin (28) [Baylor et al. Abstr. 944].

Auch in der European Collaborative Study wurden Schwangerschaftsverläufe und Nebenwirkungen im Zeitraum 1998-2002 analysiert. Die Übertragungsrate in diesem Zeitraum lag bei 3\%, wobei $88 \%$ der Schwangeren (1.252/1.415) während der Schwangerschaft antiretroviral behandelt wurden, meist mit einer PI-enthaltenden HAART. Der Anteil der geplanten Kaiserschnittentbindungen ging von $73 \%$ im Jahr 1999 auf 58\% im Jahr 2002 zurück. Zeitgleich mit dem zunehmenden Einsatz von HAART in der Schwangerschaft stieg auch die Frühgeburtlichkeit (vor HAART, d. h. von 19941997, lag die Rate bei 14\%, 1998 bei $27 \%$ und 2002 bei $31 \%$ ). Besonders stark stiegen die Rate ernster Frühgeburtlichkeit (<34 Wochen; $3 \%$ in den Jahren von 1994$1997,16 \%$ in den Jahren von 1998-2002) und extrem niedrigen Geburtsgewichtes $(<1.500$ g; $0,5 \%$ vs. $6 \%$ ). Die Neugeborenensterblichkeit stieg von 6,3/1.00o auf 13,4/1.0oo, wobei die meisten Todesfälle bei Frühgeburten zu beklagen waren [Thorne et al. Abstr. 98].

Im Unterschied zur europäischen Studie fand sich bei einer Auswertung des Antiretroviral Pregnancy Registers kein Hinweis auf eine erhöhte Frühgeburtlichkeit unter HAART-Therapie verglichen mit einer Monoprophylaxe. Allerdings wurde auch hier über eine höhere Rate an Geburten mit extrem niedrigem $\mathrm{Ge}$ burtsgewicht berichtet. Besonders gefährdet waren Schwangere, die bereits im ersten und zweiten Trimester mit einer Kombinationstherapie behandelt worden waren [Beckerman et al. Abstr. 97].

Über Fortschritte bei der Implementierung von Programmen zur Prävention der Mutter-Kind-Übertragung wurde aus 3 afrikanischen Ländern berichtet. In Zimbabwe boten im Jahr 2002 nur $69 \mathrm{Ge}$ sundheitseinrichtungen ( $5 \%$ der Gesamtzahl) Maßnahmen zur Verhütung der Mutter-Kind-Übertragung an, darunter aber mehr als die Hälfte der größeren Distriktkrankenhäuser. Von den über 45.000 Frauen, die in diesen Einrichtungen in der Schwangerschaft betreut worden waren, erhielten knapp 34.000 eine HIVTestberatung, und etwas mehr als 22.000 (49\%) wurden tatsächlich getestet. Von den getesteten Schwangeren waren $22 \%$ (4.861) mit HIV infiziert. Von diesen Frauen erhielten wiederum $35 \%$ entweder $\mathrm{Ne}$ virapin oder Zidovudin. Nur bei 1,5\% der getesteten Frauen wurde auch der Partner getestet.

Im westafrikanischen Guinea-Bissau lag die Akzeptanz des HIV-Testes bei Schwangeren, die nach Januar 2002 betreut wurden, zunächst bei $56 \%$, stieg dann aber mit Ausweitung des Programms und intensiverer Schulung des Personals auf $82 \%$. Die HIV-Prävalenz lag bei 7,6\%. In Guinea-Bissau wird HIVinfizierten Schwangeren Babynahrung zur Verfügung gestellt, sodass sie auf das Stillen verzichten können [Gianelli et al. Abstr. 897].

Aus Mosambik wurde über ein von Italien unterstütztes lokales Programm berichtet, in dem seit Mai 2002 im Rahmen der Schwangerschaftsbetreuung eine HIV-Testberatung, Ernährungsberatung und Nahrungsmittelhilfe, Gesundheitserziehung und kostenlose HAART angeboten werden. Frauen, die eine eigene Behandlungsindikation haben, werden auch nach der Entbindung unbegrenzt weiterbehandelt, Frauen ohne Behandlungsindikation bis 30 Tage nach der Entbindung. Das Testangebot wurde von 93\% der beratenen Schwangeren akzeptiert, der Anteil der HIV-Infizierten lag bei $19 \%$. Von den HIV-infizierten Schwangeren nahmen $75 \%$ die Medikamente wie empfohlen, 12,5\% lehnten eine Behandlung ab, $8 \%$ brachen die Behandlung vor der Entbindung ab, und 5\% erschienen nach der Entbindung nicht wieder. Die Mutter-Kind-Übertragungsrate lag bei 3,2\% [Liotta et al. Abstr. 901].

\section{Krankheitsverlauf bei HIV-infizierten Neugeborenen}

Bei einigen Neugeborenen wird schon sehr früh nach der Geburt mit einer antiretroviralen Kombinationstherapie begonnen. In einer Studie wurden bei 12 Kindern, die im Mittel ab dem zweiten Lebensmonat über einen Zeitraum von 3,6 Jahren erfolgreich behandelt worden waren und die kei- 
ne eigene HIV-Antikörperantwort entwickelt hatten, mit einem speziellen Protokoll versucht, Virus aus ruhenden CD4positiven Zellen zu isolieren. Bei 10 Kindern gelang die Isolierung. Dies bedeutet, dass sich trotz des frühen Behandlungsbeginns und des Ausbleibens einer Serokonversion ein latentes HIV-Reservoir etabliert hat [Persaud et al. Abstr. 387].

In einer Kohorte von 59 HIV-infizierten Kindern aus New York wurde untersucht, ob es einen Einfluss auf die Krankheitsprogression hat, wenn das Kind entscheidende HLA-Antigene von der Mutter oder vom Vater erbt. Bestimmte HLAAntigene sind mit einer verzögerten Krankheitsprogression assoziiert. Stammen diese vom Vater, verläuft auch beim Kind die Krankheitsprogression langsamer. Der Schutzeffekt dieser HLA-Antigene ist aber aufgehoben, wenn sie von der Mutter vererbt sind, da sich das Virus bereits mit der mütterlichen Immunantwort auseinander gesetzt hat [Kuhn et al. Abstr. 94]. In einer weiteren Studie zur Immunantwort HIV-infizierter Kinder konnte festgestellt werden, dass Kinder im Gegensatz zu Erwachsenen oft noch fähig sind, gegen ein mutiertes Virus (Escapevariante), das der zellvermittelten Immunantwort entkommt, eine weitere Antwort aufzubauen [Feeney et al. Abstr. 100].

Anmerkung. Dieser Bericht bezieht sich größtenteils auf die Zusammenfassung der Konferenzvorträge, die auf der offiziellen Webseite der Konferenz unter http://www.retroconference.org/2004/ AbstractSearch/AbstractSearch.aspx auffindbar sind.

\section{Korrespondierender Autor \\ Dr.U.Marcus}

Robert Koch-Institut, Nordufer 20, 13353 Berlin

E-Mail: marcusu@rki.de

\section{Literatur}

1. Porco TC, Martin JN, Page-Shafer KA et al. (2004) Decline in HIV infectivity following the introduction of highly active antiretroviral therapy. AIDS 18:81-88

2. Heringlake S, Ockenga J, Tillmann HL et al. (1998) GB virus $C /$ hepatitis $G$ virus infection: a favorable prognostic factor in human immunodeficiency virus-infected patients? J Inf Dis 177:1723-1726
3. Lefrère JJ, Roudot-Thoraval F, Morand-Joubert et al. (1999) Carriage of GB virus C/ hepatitis G virus RNA is associated with a slower immunologic, virologic, and clinical progression of human immunodeficiency virus disease in coinfected persons. J Inf Dis 179:783-789

4. Toyoda H, Fukuda Y, Hayakawa T et al. (1998) Effect of $G B$ virus $C /$ hepatitis $G$ virus coinfection on the course of HIV infection in hemopühilia patients in Japan.J Acquir Immune Defic Syndr Hum Retrovirol 17:209-213

5. Yeo AET, Matsumoto A, Hisada M et al. (2000) Effect of hepatitis $G$ virus infection on progression of HIV infection in patients with hemophilia. Ann Intern Med 132:959-963

6. Birk M, Lindbäck S, Lidman C (2002) No influence of GB virus $C$ replication on the prognosis in a cohort of HIV-1 infected patients. AIDS 16:2482-2485

7. Brust D, Jagannatha S, Herpin B et al. (2002) Hepatitis $G$ virus infection does not prolong survival of patients with early-stage HIV disease: importance of baeline HIV viral load as a predictor of mortality. Abstr.WeOrC1378, Internationale AIDS-Konferenz, Barcelona

8. Sabin CA, Devereux H, Kinson Z et al. (1998) Effect of coinfection with hepatitis $\mathrm{G}$ virus on HIV disease progression in hemophilic men.J Acquir Immune Defic Syndr Hum Retrovirol 19:546-547

9. Xiang J,Wünschmann S, Diekema DJ et al. (2001) Effect of coinfection with GB virus $C$ on survival among patients with HIV infection. N Eng J Med 345:707-714

10. Tillmann HL, Heiken $H$, Knapik-Botor A et al. (2001) Infection with $G B$ virus $C$ and reduced mortality among HIV-infected patients. N Eng J Med 345:715-724

11. Williams CF, Klinzman D, Yamashita TE et al. (2004) Persistent $G B$ virus $C$ infection and survival in HIV-infected men. N Eng J Med 350:981-990

12. Van der Bij A, Kloosterboer N, Prins M et al. (2004) Loss of $G B$ virus $($ viremia over time is associated with faster progression among HIV+ homosexual men with an imputed date of seroconversion. Abstr. 806, 11th Conference on Retroviruses and Opportunistic Infections, San Francisco

13. Björkman P,Flamholc L, Naucler A et al. (2004) GB virus C during the natural course of HIV-1 infection: viremia at diagnosis does not predict mortality. AIDS 18:877886

14. Gottlieb GS, Nickle DC, Jensen MA et al. (2004) Dual HIV1 infection associated with rapid disease progression. Lancet 363:619-622

\section{IKOP-Preis für angewandte Infektionsprävention}

Postoperative Wundinfektionen sind nach wie vor eine bedeutende Krankheitsursache im Klinikalltag und nehmen unter den nosokomialen Infektionen den dritten Platz ein. Nicht selten ist die Behandlung der Infektion kostenintensiver als der eigentliche operative Eingriff und verlängert den Krankenhausaufenthalt erheblich. Dennoch werden diese Infektionen häufig nicht erfasst bzw. als nicht vermeidbar toleriert.

Zur Unterstützung der wissenschaftlichen Forschungsarbeit auf dem Gebiet der Infektionskontrolle schreibt daher der Expertenkreis IKOP (Infektionskontrolle im OP) erstmalig den „IKOP-Innovationspreis für angewandte Infektionsprävention" aus. Die Vergabe richtet sich an Wissenschaftler, die auf dem Gebiet der nosokomialen Infektionen Errungenschaften vorweisen können (Mediziner, Biologen, Pharmazeuten etc.). Es sollen Forschungsarbeiten prämiert werden, die Innovationen für den klinischen Alltag versprechen. Nominierungsgrundlage bilden Arbeiten von hoher wissenschaftlicher Qualität, die aktuell, im Forschungsansatz originell und bedeutend für den medizinischen Fortschritt sind.

Die Auszeichnung wird einmal jährlich ausgeschrieben und mit einem Honorar von EUR 5000 ausgelobt. Eingereicht werden können wissenschaftliche Arbeiten, die zum Zeitpunkt der Einreichung entweder noch nicht oder maximal in den letzten zwölf Monaten veröffentlicht wurden. Über die Vergabe entscheidet die Jury der IKOP-Mitglieder. Die Preisverleihung findet anlässlich des 6. Ulmer Symposiums „Krankenhausinfektionen“ im April 2005 statt. Abgabetermin ist der 31. Januar 2005. Die Bewerbung ist in dreifacher Ausfertigung unter Beifügung des Lebenslaufs und eines wissenschaftlichen Tätigkeitsberichts einzureichen. Einsendungen erfolgen an: PD Dr. Sebastian Lemmen Universitätsklinikum Aachen Zentralbereich für Krankenhaushygiene Pauwellstr. 30, 51074 Aachen

Tel.: 0241/8089-843,-884

Fax: 0241/8020-540

E-mail: slemmen@ukaachen.de

Quelle: Expertenkreis Infektionskontrolle im OP (IKOP) 